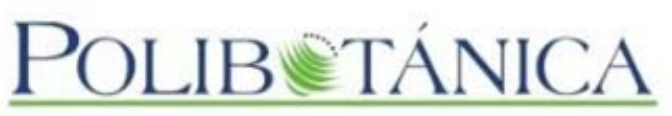

\title{
Polibotánica
}

ISSN electrónico: 2395-9525

polibotanica@gmail.com

Instituto Politécnico Nacional

México

http://www.polibotanica.mx

\section{ACTIVIDAD ANTIMICROBIANA in vitro DE EXTRACTOS DE Jatropha dioica Seseé CONTRA BACTERIAS FITOPATÓGENAS DE TOMATE.}

\section{In vitro ANTIMICROBIAL ACTIVITY OF Jatropha dioica Seseé EXTRACTS AGAINST TOMATO PHYTO-PATHOGENIC BACTERIA.}

Pérez-Pérez, J.U., D. Guerra-Ramírez, B. Reyes-Trejo, J.A. Cuevas-Sánchez y P. GuerraRamírez.

ACTIVIDAD ANTIMICROBIANA in vitro DE EXTRACTOS DE Jatropha dioica Seseé CONTRA BACTERIAS FITOPATÓGENAS DE TOMATE.

In vitro ANTIMICROBIAL ACTIVITY OF Jatropha dioica Seseé EXTRACTS AGAINST TOMATO PHYTO-PATHOGENIC BACTERIA.

POLIBETANICA

Instituto Politécnico Nacional
Núm. 49: 125-133 México. Enero 2020

DOI: $10.18387 /$ polibotanica. 49.8

(C) (i) Este es un artículo de acceso abierto bajo la licencia Creative Commons 4.0 Atribución-No Comercial (CC BY-NC 4.0 Internacional). 


\section{ACTIVIDAD ANTIMICROBIANA in vitro DE EXTRACTOS DE Jatropha dioica Seseé CONTRA BACTERIAS FITOPATÓGENAS DE TOMATE.}

\section{In vitro ANTIMICROBIAL ACTIVITY OF Jatropha dioica Seseé EXTRACTS AGAINST TOMATO PHYTO-PATHOGENIC BACTERIA.}

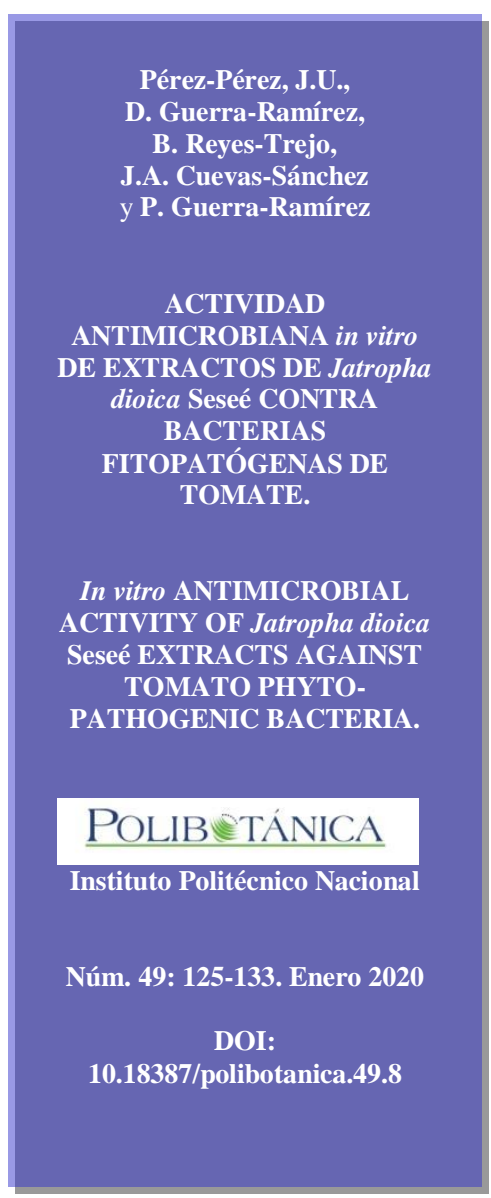

Pérez-Pérez, J.U.,

Reyes-Trejo,

J.A. Cuevas-Sánchez

MICROBIANA in vitro

a Seseé CONTRA

PATÓGENAS DE

TOMATE.

In vitro ANTIMICROBIAL

ACTIVITY OF Jatropha dioica

TOMATO PHYTO-

TENIC BACTERIA.

POLIBETÁNICA

Instituto Politécnico Nacional

Núm. 49: 125-133. Enero 2020

DOI:

10.18387/polibotanica.49.8

\author{
J.U. Pérez-Pérez \\ Departamento de Fitotecnia, Universidad Autónoma Chapingo.
}

D. Guerra-Ramírez

B. Reyes-Trejo

Laboratorio de Productos Naturales, Área de Química,

Departamento de Preparatoria Agrícola, Universidad Autónoma Chapingo Carretera Federal México-Texcoco Km 38.5, 56230 Texcoco, Méx.

J.A. Cuevas-Sánchez

Banco de Germoplasma, Departamento de Fitotecnia, Universidad Autónoma Chapingo.

P. Guerra-Ramírez / pris682000@yahoo.com.mx Laboratorio de Productos Naturales, Área de Química, Departamento de Preparatoria Agrícola, Universidad Autónoma Chapingo Carretera Federal México-Texcoco Km 38.5, 56230 Texcoco, Méx.

RESUMEN: Se utilizó el método de microdiluciones en microplaca para evaluar la inhibición del crecimiento in vitro de las bacterias fitopatógenas Clavibacter michiganensis subsp. michiganensis $(\mathrm{Cmm})$, Pseudomonas syringe $\mathrm{pv}$. tomato $(\mathrm{Pst}) \mathrm{y}$ Xanthomonas campestris pv. vesicatoria $(\mathrm{Xcv})$ con extractos de diferente polaridad obtenidos a partir de raíces de Jatropha dioica Seseé (Sangre grado), especie vegetal endémica de México valorada en la medicina tradicional por sus propiedades farmacológicas y curativas. Como indicador de respiración celular en cultivos bacterianos expuestos a extractos, se utilizó cloruro de 2,3,5-trifeniltetrazolio. Los extractos metanólico, hexánico y de acetato de etilo, presentaron actividad antimicrobiana contra los fitopatógenos Cmm, Pst y Xcv. De acuerdo con los resultados, el extracto hexánico mostró la menor concentración Inhibitoria Media Máxima ( $\left.\mathrm{IC}_{50}\right)$ contra Pst y Xcv $\left(0.5 \pm 0.01\right.$ y $1.7 \pm 0.36 \mathrm{mg} \mathrm{mL}^{-1}$, respectivamente) por lo que se separó por cromatografía en columna. Como resultado, se aisló y purificó un sólido cristalino blanco identificado espectroscópicamente como citlalitriona, dicho compuesto también presentó actividad contra las bacterias $\mathrm{Cmm}$, Pst y Xcv con una $\mathrm{CI}_{50}$ de $1.0 \pm 0.13,1.0 \pm 0.11$ y $1.1 \pm 0.19 \mathrm{mg} \mathrm{mL}^{-1}$, respectivamente. Estos resultados in vitro abren la posibilidad de utilizar extractos vegetales de $J$. dioica para el control de las enfermedades causadas por las bacterias estudiadas en el cultivo de tomate, ofreciendo una alternativa al uso de antibióticos o compuestos de cobre.

Palabras clave: extractos vegetales, citlalitriona, antibacterianos, fitopatógenos, Solanum lycopersicum, Euphorbiaceae, Sangre Grado.

ABSTRACT: The microplate microdilution method was used to evaluate the inhibition of in vitro growth of Clavibacter michiganensis subsp michiganensis $(\mathrm{Cmm})$, Pseudomonas syringe pv. tomato (Pst) and Xanthomonas campestris pv. 
vesicatory $(\mathrm{Xcv})$ phytopathogenic bacteria, with extracts of different polarity obtained from roots of Jatropha dioica Seseé (Blood grade), endemic plant species of Mexico valued in traditional medicine for its pharmacological and healing properties. As an indicator of cellular respiration in bacterial cultures exposed to extracts, 2,3,5-triphenyltetrazolium chloride was used. The methanolic, hexane and ethyl acetate extracts showed antimicrobial activity against the phytopathogens Cmm, Pst and Xcv. According to the results, the hexane extract showed the lowest Maximum Average Inhibitory concentration $\left(\mathrm{IC}_{50}\right)$ against Pst and $\mathrm{Xcv}(0.5 \pm 0.01$ and $1.7 \pm 0.36 \mathrm{mg} \mathrm{mL}^{-1}$, respectively), so it was separated by column chromatography. As a result, a white crystalline solid identified spectroscopically as citlalitrione was isolated and purified, said compound also exhibited activity against the $\mathrm{Cmm}$, Pst and $\mathrm{Xcv}$ bacteria with an $\mathrm{IC}_{50}$ of $1.0 \pm$ $0.13,1.0 \pm 0.11$ and $1.1 \pm 0.19 \mathrm{mg} \mathrm{mL}^{-1}$, respectively. These in vitro results open the possibility of using plant extracts of J. dioica for the control of diseases caused by the bacteria studied in tomato cultivation, offering an alternative to the use of antibiotics or copper compounds.

Keywords: plant extracts, citlalitrione, antibacterials, phytopathogens, Solanum lycopersicum, Euphorbiaceae, Sangre Grado.

\section{INTRODUCCIÓN}

Las enfermedades bacterianas son un problema grave en sistemas de producción en invernadero y a campo abierto. Entre los principales patógenos bacterianos responsables del daño en órganos de tomate como raíces, tallos, ramas, hojas, brotes, flores y frutos en las regiones cálidas y templadas del mundo se encuentran las tres bacterias que se emplearon en este trabajo: Pseudomonas syringae pv. tomato, agente causal de la peca bacteriana que provoca la deformación de los frutos, los cuales aparecen con manchitas deprimidas o manchas pequeñas y sobresalientes, asimismo, esta enfermedad provoca la caída de las flores, lo cual puede reducir en un $75 \%$ la cosecha. Por otro lado, Xanthomonas vesicatoria, es una bacteria que afecta hojas, tallos y frutos, y causa manchas de 2 a $3 \mathrm{~mm}$ que se vuelven angulares y cuya superficie puede parecer grasienta, con el centro traslúcido y el borde negro. Finalmente, Clavibacter michiganensis subsp. michiganensis es el agente causal del cancro bacteriano, enfermedad muy seria en tomate, distribuida a nivel mundial, afecta los frutos, provocando manchas oscuras rodeadas de un halo blanco, similar a un ojo de pájaro (López-Marín, 2017). Las dos primeras bacterias causan enfermedades parenquimáticas, mientras que la última causa enfermedades parenquimáticas y vasculares (Balestra, Heydari, Ceccarelli, Ovidi, \& Quattrucci, 2009).

En la actualidad, solo se dispone de algunas medidas para el control de las enfermedades mencionadas, en particular en la agricultura orgánica. Los principales problemas en el control de enfermedades bacterianas son la falta de disponibilidad de compuestos antibacterianos comerciales adecuados. Los antibióticos son cuestionables por varias razones y, por lo tanto, están prohibidos en muchos países. Los tratamientos con cobre y las prácticas agronómicas apropiadas, como la certificación de semillas, el riego y la fertilización, son las principales medidas utilizadas actualmente para controlar las enfermedades mencionadas. Como alternativa a los compuestos de cobre, recientemente se han propuesto algunas sustancias naturales, pero se necesitan más estudios para optimizar su efectividad. Considerando la falta de alternativas válidas a los compuestos de cobre y la falta de disponibilidad de cultivares comerciales resistentes a Pst, $\mathrm{Xcv}$ y $\mathrm{Cmm}$, se ha propuesto como alternativa el uso de extractos vegetales y/o sus metabolitos secundarios (Handelsman \& Stabb, 1996).

De acuerdo con (Cowan, 1999), entre los principales grupos de metabolitos secundarios presentes en las plantas con propiedades antimicrobianas se encuentran los compuestos fenólicos, algunos terpenoides presentes en los aceites esenciales y los alcaloides que son compuestos nitrogenados heterocíclicos.

J. dioica perteneciente a la familia Euphorbiaceae, es una de las 199 especies hasta ahora registradas y una de las 48 especies endémicas de México (Steinmann, 2002). J. dioica es una 
planta que crece en zonas áridas del noreste de México y es empleada en la medicina tradicional preparada como decocción para el control de caída de cabello, dolor de riñones y problemas de digestión (Manzanero-Medina, Flores-Martínez, Sandoval-Zapotitla, \& Bye-Boettler, 2009). Se tienen registros de su actividad antiviral (Silva-Mares et al., 2013) y antimicrobiana contra patógenos humanos (Silva-Belmares, Rivas-Morales, Viveros-Valdez, de la Cruz-Galicia, \& Carranza-Rosales, 2014) además, se han demostrado sus propiedades antioxidantes y quimioprotectoras (Martínez et al., 2014). Actualmente se han identificado tres diterpenos con actividad biológica presentes en $J$. dioica: riolozatriona (Dominguez et al., 1980) citlalitriona y jatrophona (Villarreal, Dominguez, Williams, Scott, \& Reibenspies, 1988), además de algunos compuestos como metabolitos secundarios propios de las plantas como flavonoides y polifenoles (Silva-Belmares et al., 2014) que posiblemente son los responsables de las propiedades curativas de la planta. Sin embargo, para el caso del diterpeno citlalitriona, no se ha evaluado su bioactividad.

El objetivo de este trabajo fue evaluar mediante el método de microdiluciones en microplaca la actividad antimicrobiana de extractos de diferente polaridad y en su caso, metabolitos secundarios obtenidos de raíces de J. dioica, contra las bacterias fitopatógenas Clavibacter michiganensis subsp. michiganensis (Cmm), Pseudomonas syringe pv. tomato (Pst), Xanthomonas campestris pv. vesicatoria (Xcv).

\section{MATERIAL Y MÉTODOS}

\section{Bacterias fitopatógenas}

Las cepas bacterianas fueron proporcionadas por Departamento de Fitosanidad-Fitopatología del Colegio de Postgraduados, Campus Montecillo, Estado de México. Las bacterias Ccm, Pst y $\mathrm{Xcv}$ se cultivaron en caldo nutritivo BIOXON® (extracto de carne $3 \mathrm{~g} \mathrm{~L}^{-1}$, peptona de carne $5 \mathrm{~g}$ $\mathrm{L}^{-1}, \mathrm{pH}=7 \pm 0.2$ ) a $28^{\circ} \mathrm{C}$ y $110 \mathrm{rpm}$ hasta obtener una absorbancia de 0.5 a $600 \mathrm{~nm}$. Se mantuvieron en refrigeración a $4^{\circ} \mathrm{C}$ hasta su uso.

\section{Material vegetal}

Las raíces de $J$. dioica fueron colectadas en la localidad de Buenavistilla, Municipio de San José Iturbide, Guanajuato, México en junio-julio 2017. Un ejemplar fue identificado y depositado en el Herbario-Hortorio "Jorge Espinoza Salas" del departamento de Preparatoria Agrícola de la Universidad Autónoma Chapingo. Las raíces se dejaron secar a la sombra, después se cubrieron y resguardaron a temperatura ambiente hasta su procesamiento.

\section{Obtención de extractos de raíces de $\boldsymbol{J}$. dioica}

Las raíces de $J$. dioica previamente secas y molidas $(9 \mathrm{~kg}$ ) se maceraron en metanol por 120 horas. El extracto metanólico se concentró en un rota-evaporador marca Buchi modelo R-210 a una temperatura de $50^{\circ} \mathrm{C}$. Una porción del extracto se disolvió en agua en una proporción 1:20 (p/v) y se extrajo sucesivamente con $n$-hexano y acetato de etilo, después de evaporar los disolventes, se calculó el rendimiento obtenido de cada uno de los extractos.

Una porción del extracto hexánico $(8.6 \mathrm{~g})$, se fraccionó por cromatografía en columna empacada con gel de sílice y se eluyó con mezclas de hexano-AcOEt de polaridad creciente (10:0, 9:1, 4:1, 5:5, 0:10). En las fracciones eluídas con hexano-AcOEt en proporción 4:1 se observó la presencia de un sólido blanco, el cual fue sometido a una nueva separación por cromatografía en columna, colectándose 49 fracciones de $6 \mathrm{~mL}$ cada una. Las fracciones 29-32 contenían $\beta$-sitoesterol, lo cual se determinó comparando con una muestra auténtica: en las fracciones 33-37 se encontró otro sólido blanco, el cual fue identificado como citlalitriona por los métodos espectroscópicos de infra-rojo y resonancia magnética nuclear de hidrógeno y carbono 13. Cada uno de estos metabolitos secundarios fue evaluado para determinar su actividad antimicrobiana contra las tres cepas bacterianas. 
Para las pruebas antimicrobianas, se disolvieron $0.1 \mathrm{~g}$ de extracto o los metabolitos purificados en $20 \mathrm{~mL}$ de dimetilsulfoxido al 10\% (Salie, Eagles, \& Leng, 1996), para obtener una concentración final de $5 \mathrm{mg} \mathrm{mL}^{-1}$. Las mezclas se esterilizaron con filtros para jeringa de 0.45 $\mu \mathrm{m}$ y se resguardaron en frascos ámbar a $4^{\circ} \mathrm{C}$ hasta su uso.

\section{Evaluación antimicrobiana por método de microdilución en microplaca.}

La actividad antimicrobiana de los extractos o metabolitos secundarios, se probó por el método de microdilución en microplaca de acuerdo a lo descrito por Das, Tiwari, \& Shrivastava (2010). En cada pozo de una microplaca se mezcalron $50 \mu \mathrm{L}$ de cada uno de los cultivos bacterianos de $\mathrm{Ccm}$, Pst y Xcv, a una absorbancia de 0.5 a $600 \mathrm{~nm}$ (equivalente a $1 \times 10^{6}$ unidades formadoras de colonias por mililitro); el extracto vegetal en diluciones seriadas de 200 a $20 \mu \mathrm{L}$ (escala de $20 \mu \mathrm{L}$ ), y el medio de cultivo estéril de 0 a $180 \mu \mathrm{L}$ (escala de $20 \mu \mathrm{L}$ ), para obtener un volúmen final de $250 \mu \mathrm{L}$. Como control negativo se utilizó medio de cultivo $(200 \mu \mathrm{L})$ y la suspensión bacteriana $(50 \mu \mathrm{L})$, como control positivo se utilizó el medio de cultivo $(150 \mu \mathrm{L})$, la suspensión bacteriana $(50 \mu \mathrm{L})$ y etanol grado reactivo $(50 \mu \mathrm{L})$. Las microplacas se incubaron a $37^{\circ} \mathrm{C}$ por $24 \mathrm{~h}$. Posteriormente, en cada pozo se adicionaron $20 \mu \mathrm{L}$ de 2,3,5-cloruro de tetrazolio (CTT) al $2 \%$ y se incubó a $37^{\circ} \mathrm{C}, 110 \mathrm{rpm}$ por 60 minutos. Finalmente se midió la absorbancia a $540 \mathrm{~nm}$ en un lector de microplacas Sinergy 2 marca BioTek (Gabrielson et al., 2002) controlado por una interfaz de análisis de datos Gen5. El porcentaje de inhibición bacteriana se calculó con la Ecuación 1, posteriormente se graficó las concentraciones de extracto contra el porcentaje de inhibición bacteriana obteniendo así una ecuación de la recta mediante una regresión lineal.

Porcentaje de inhibicion bacteriana $=\frac{(\mathrm{Ac}-\mathrm{Ae})}{\mathrm{Ac}} \mathrm{x} 100 \ldots \ldots \ldots \ldots \ldots \ldots \ldots$. Ecuación 1

Donde:

$\mathrm{Ac}=$ absorbancia del control negativo y $\mathrm{Ae}=$ absorbancia del ensayo.

Se preparó también una placa para ser usada como blanco en la medición de la absorbancia, de la manera descrita anteriormente pero con $100 \mu \mathrm{l}$ de caldo nutritivo (sin cultivo bacteriano).

Para calcular la Concentración Inhibitoria Media Máxima $\left(\mathrm{CI}_{50}\right)$

Se graficó la concentración de extracto vs el porcentaje de inhibición. Con la ecuación de la recta obtenida se calculó la concentración a la que la inhibición del crecimiento bacteriano fue del 50\%, para obtener así la $\mathrm{CI}_{50}$, mediante la ecuación de regresión considerando coeficientes de correlación $\left(\mathrm{R}^{2}\right)$ mayores a 0.90 .

Los resultados se expresaron como medias \pm desviación estándar. Cada ensayo se efectuó por triplicado.

\section{RESULTADOS}

La efectividad antimicrobiana de los extractos expuestos a bacterias Cmm, Pst y Xcv se muestran en el cuadro 1. El extracto hexánico y de acetato de etilo, muestran actividad antimicrobiana contra las tres cepas evaluadas, no así para el extracto metanólico que sólo tuvo respuesta para Pst y Xcv.

La mejor respuesta antimicrobiana se observó con el extracto hexánico contra Pst y Xcv respectivamente y con el extracto acetato de etilo contra $\mathrm{Cmm}$. 
Cuadro 1. Concentración Inhibitoria Media Máxima $\left(\mathrm{CI}_{50}\right)$ de extractos vegetales de $J$. dioica contra bacterias fitopatógenas expresado en $\mathrm{mg} \mathrm{mL}^{-1}$.

\begin{tabular}{lccc}
\hline \multirow{2}{*}{ Extracto } & \multicolumn{3}{c}{$\mathbf{C I}_{\mathbf{5 0}}\left(\mathbf{m g ~ m}_{\mathbf{~ m}}^{\mathbf{- 1}}\right)$} \\
\cline { 2 - 4 } & $\mathbf{C m m}$ & Pst & $\mathbf{X c v}$ \\
\hline Hexánico & $4.4 \pm 0.92$ & $0.5 \pm 0.01$ & $1.7 \pm 0.36$ \\
Acetato de etilo & $2.0 \pm 0.43$ & $3.5 \pm 0.73$ & $2.3 \pm 0.52$ \\
Metanólico & $(-)$ & $5.8 \pm 1.12$ & $2.9 \pm 0.68$
\end{tabular}

(-): sin actividad antimicrobiana; Cmm: Clavibacter michiganensis subsp. michiganensis; Pst: Pseudomonas syringe pv. tomato; $\mathrm{Xcv}$ : Xanthomonas campestris pv. vesicatoria.

Debido a que el extracto hexánico mostró una alta efectividad antimicrobiana contra Pst y Xcv $\left(\mathrm{CI}_{50}=0.5\right.$ y $1.7 \mathrm{mg} \mathrm{mL}^{-1}$, respectivamente), fue sometió a un fraccionamiento por cromatografía en columna utilizando $300 \mathrm{~mL}$ de los siguientes eluyentes: $n$-hexano, mezclas de AcOEt-hexano de polaridad creciente $(9: 1$, , $4: 1$ y 1:1 v/v) y AcOEt, las fracciones obtenidas (F1,F2, F3, F4, y F5) fueron evaluadas para determinar su actividad antimicrobiana, y se encontró que F3 presentaba una mayor actividad antimicrobiana, por lo que se separó por cromatografía en columna utilizando como eluyente mezclas de hexano/AcOEt de polaridad creciente y obteniendo un total de 42 fracciones. En las fracciones eluídas con una mezcla de hexano/AcOEt (4:1) se observó un sólido, resultando la fracción F'1 a la que se les denominó como aguas madres, y una muestra sólida cristalina a la que se le distinguió como F'2, identificada como citlilitriona. Finalmente las fracciones eluidas con la mezcla de hexano:acetato de etilo (5:5) se reunieron en la fracción nombrada como F'3. En el cuadro 2, se puede observar que tanto las aguas madres de la fracción F'1 como la citlalitriona de la fracción F'2, mostraron valores de $\mathrm{CI}_{50}=0.1 \mathrm{mg} \mathrm{mL}^{-1}$ contra las tres bacterias fitopatogenas $\mathrm{Cmm}$, Pst y $\mathrm{Xcv}$, en tanto que la fracción F'3 mostró valores de inhibición relativamente superiores sobre las tres bacterias, sin embargo, al observar su composición por cromatografía en capa delgada, se detectó que correspondía a una mezcla compleja de metabolitos secundarios que no se analizó.

Cuadro 2. Concentración Inhibitoria Media Máxima $\left(\mathrm{CI}_{50}\right)$ de fracciones hexánicas de $J$. dioica contra bacterias fitopatógenas expresado en $\mu \mathrm{L} \mathrm{mL}^{-1}$.

\begin{tabular}{lccc}
\hline \multirow{2}{*}{ Fracción } & \multicolumn{3}{c}{ CI50 $\left(\mu \mathbf{L} \mathbf{~ m L}^{-1}\right)$} \\
\cline { 2 - 4 } & Cmm & Pst & Xcv \\
\hline F'1 & $0.9 \pm \underline{0.09}$ & $1.0 \pm \underline{0.14}$ & $0.6 \pm \underline{0.02}$ \\
F'2 & $1.0 \pm \underline{0.13}$ & $1.0 \pm \underline{0.11}$ & $1.1 \pm \underline{0.19}$ \\
F'3 & $1.2 \pm \underline{0.21}$ & $1.1 \pm \underline{0.2}$ & $0.7 \pm \underline{0.01}$
\end{tabular}

F'1: aguas madre; F'2: citlalitriona; F'3: mezcla compleja de metabolitos secundarios; Cmm: Clavibacter michiganensis subsp. michiganensis; Pst: Pseudomonas syringe pv. tomato; Xcv: Xanthomonas campestris pv. vesicatoria. 
$\mathrm{Al}$ sólido cristalino de la fracción F'2, se le determinó su punto de fusión (201-203 $\left.{ }^{\circ} \mathrm{C}\right)$, además su espectro de infrarrojo mostró bandas en 1746, 1692, 1446, 1388.5, 1347, 1156.9 y 1038.7 $\mathrm{cm}^{-1}$, que se asignaron a grupos carbonilo de cetona $\left(\mathrm{R}_{2} \mathrm{C}=\mathrm{O}\right)$, de al menos un enlace doble $(-\mathrm{C}=\mathrm{C}-)$ y de enlace C-O-C tipo éter. En su espectro de Resonancia Magnética Nuclear de ${ }^{1} \mathrm{H}$ (RMN- ${ }^{1} \mathrm{H}$., $400 \mathrm{MHz}, \mathrm{CDCl}_{3}$ ), se observaron los siguientes desplazamientos químicos $\delta: 3.30$ (s, H3), 5.51 (q, $J=1.6, \mathrm{H} 5), 2.95(\mathrm{dd}, J=12.7,1.8, \mathrm{H} 8 \alpha), 2.60(\mathrm{dd}, J=12.7,13.13, \mathrm{H} 8 \beta), 3.66$ (dd, $J=9.5,1.5, \mathrm{H} 15), 1.15\left(\mathrm{CH}_{3}, \mathrm{~d}, J=7.5, \mathrm{H} 16\right), 1.95\left(\mathrm{CH}_{3}, \mathrm{~d}, J=1.6, \mathrm{H} 17\right), 1.30\left(\mathrm{CH}_{3}, \mathrm{~s}, \mathrm{H} 18\right)$ $0.97\left(\mathrm{CH}_{3}, \mathrm{~s}, \mathrm{H} 19\right), 1.44\left(\mathrm{CH}_{3}, \mathrm{~s}, \mathrm{H} 20\right)$ : en el espectro de Resonancia Magnética Nuclear de ${ }^{13} \mathrm{C}$ (RMN- ${ }^{13} \mathrm{C}$., $100 \mathrm{MHz}, \mathrm{CDCl}_{3}$ ), se observaron 20 señales, cuyos desplazamientos químicos $(\delta)$ fueron: 217 (C-12), 215.3 (C-14), 208.2 (C-7), 145.4 (C-6), 128.2 (C-5), 77.2 (C-3), 67.5 (C-4), 65.6 (C-13), 55.4 (C-11), 52.8 (C-9), 46.5 (C-15), 37.9 (C-8), 37.5 (C-10), 34.8 (C-1), 33.7 (C2), 28.2 (C-18), 23.6 (C-19), 20.4 (C-17), 16.0 (C-16) y 14.5 (C-20); el análisis de los datos anteriores corresponden a un diterpenoide cuya estructura molecular fue identificada como citlalitriona (1) (Melchor-Martínez et al., 2017; Villarreal et al., 1988).

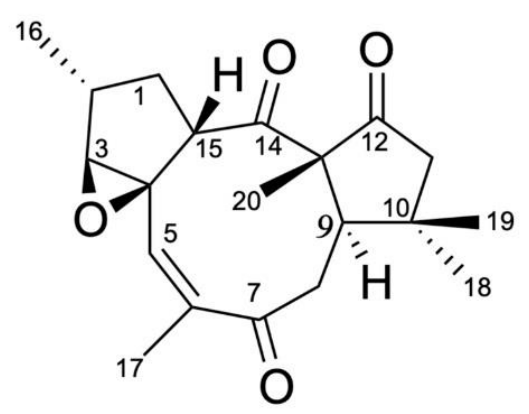

Citlalitriona (1)

\section{DISCUSIÓN}

Estudios previos, revelaron que el extracto metanólico de $J$. dioica tiene actividad antimicrobiana para Staphylococcus aureus, Klebsiella pneumoniae, Pseudomonas aeruginosa, Escherichia coli (Serrano-Gallardo et al., 2017) Porphyromonas gingivalis (Waizel-Bucay \& Martínez Rico, 2011) Bacillus cereus, Salmonella typhi, Candida labicans (Silva-Belmares et al., 2014) y antiviral (Silva-Mares et al., 2013); coincidiendo con lo encontrado en el presente trabajo en cuanto a la participación de terpenos, esteroles y metilesteroles como compuestos activos.

Por otro lado, algunos estudios señalan que los principales compuestos de un extracto son los responsables de su actividad biológica, mientras que otros concluyen que algunos componentes actúan sinérgicamente (Pitarokili, Tzakou, Loukis, \& Harvala, 2003; Bakkali \& Idaomar, 2008). Debido a que los extractos de plantas contienen una mezcla de varios componentes, es probable que su actividad antimicrobiana no sea atribuible a un solo mecanismo de acción, pero se sabe que los terpenos como la citlalitriona tienen la habilidad de dañar biomembranas debido a sus características lipofílicas (Lucini, Zunino, Lopez, \& Zygadlo, 2006).

Los resultados obtenidos sugieren que los extractos no son selectivos a bacterias gram negativas y positivas. La actividad antimicrobiana del extracto hexánico podría atribuirse principalmente al diterpeno citlalitriona. Estos resultados dan soporte científico y abren la posibilidad para el uso de J. dioica en el control de las enfermedades producidas por las bacterias fitopatógenas Cmm, Pst y Xcv. 


\section{CONCLUSIONES}

Los extractos de $J$. dioica presentaron actividad inhibitoria in vitro sobre el crecimiento de bacterias fitopatógenas que afectan al cultivo de tomate.

El extracto hexánico mostró mayor actividad antimicrobiana contra Pst y Xcv y el extracto de acetato de etilo contra $\mathrm{Cmm}$.

Del extracto hexánico se aisló e identificó el compuesto diterpénico citlalitriona como uno de los responsables de la actividad inhibitoria.

Estos resultados abren la posibilidad de utilizar estos extractos vegetales y el compuesto citlalitriona para el control de las enfermedades causadas por $\mathrm{Ccm}$, Pst y Xcv como una alternativa al uso de antibióticos o compuestos de cobre para el control de enfermedades bacterianas del cultivo de tomate (Solanum lycopersicum).

\section{AGRADECIMIENTOS}

Los autores desean agradecer el financiamiento del Consejo Nacional de Ciencia y Tecnología (CONACYT), así como al Laboratorio de Productos Naturales de la Universidad Autónoma Chapingo por las facilidades y recomendaciones hechas durante la fase experimental.

\section{LITERATURA CITADA}

Bakkali, F., \& Idaomar, M. (2008). Biological effects of essential oils - A review, 46, 446-475. https://doi.org/10.1016/j.fct.2007.09.106

Balestra, G. M., Heydari, A., Ceccarelli, D., Ovidi, E., \& Quattrucci, A. (2009). Antibacterial effect of Allium sativum and Ficus carica extracts on tomato bacterial pathogens. Crop Protection, 28(10), 807-811. https://doi.org/10.1016/j.cropro.2009.06.004

Cowan, M. M. (1999). Plant Products as Antimicrobial Agents, 12(4), 564-582.

Das, K., Tiwari, R. K. S., \& Shrivastava, D. K. (2010). Techniques for evaluation of medicinal plant products as antimicrobial agent : Current methods and future trends, 4(2), 104111. https://doi.org/10.5897/JMPR09.030

Dominguez, X. A., Cano, G., Franco, R., Villarreal, A. M., Watson, W. H., \& Zabel, V. (1980). Riolozatrione, a new class of diterpene from Jatropha dioica var. sessiliflora. Phytochemistry, 19(11), 2478. https://doi.org/10.1016/S0031-9422(00)91059-8

Gabrielson, J., Hart, M., Jarelo, A., Ku, I., Mckenzie, D., \& Mo, R. (2002). Evaluation of redox indicators and the use of digital scanners and spectrophotometer for quantification of microbial growth in microplates, 50, 63-73.

Handelsman, J., \& Stabb, E. V. (1996). Biocontrol of Soilborne Plant Pathogens, 8(October), $1855-1869$.

López-Marín, L. M. (2017). Manual técnico del cultivo de tomate. (L. Ramírez Cartin, Ed.). Costa Rica: Instituto Nacional de Innovación y Transferencia en Tecnología Agropecuaria. Retrieved from http://repositorio.iica.int/bitstream/11324/3143/1/BVE17079148e.pdf

Lucini, E. I. M. P., Zunino, M. L., Lopez, M. L., \& Zygadlo, J. A. (2006). Effect of Monoterpenes on Lipid Composition and Sclerotial Development of. $J$. Phytopathology, 154, 441-446.

Manzanero-Medina, G. I., Flores-Martínez, A., Sandoval-Zapotitla, E., \& Bye-Boettler, R. (2009). Etnobotánica de siete raíces medicinales en el mercado de Sonora de la Ciudad de México. Polibotanica, (27), 191-228.

Martínez, N., Almaguer, G., Vázquez-Alvarado, P., Figueroa, A., Zúñiga, C., \& HernándezCeruelos, A. (2014). Análisis fitoquímico de Jatropha dioica y determinación de su 
Recibido:

23/marzo/2019

Aceptado:

12/diciembre/2019 efecto antioxidante y quimioprotector sobre el potencial genotóxico de ciclofosfamida, daunorrubicina y metilmetanosulfonato evaluado mediante el ensayo cometa. Boletín Latinoamericano Y Del Caribe de Plantas Medicinales Y Aromáticas, 13(5), 437-457.

Melchor-Martínez, E. M., Silva-Mares, D. A., Torres-López, E., Waksman-Minsky, N., Pauli, G. F., Chen, S. N., ... Rivas-Galindo, V. M. (2017). Stereochemistry of a Second Riolozane and Other Diterpenoids from Jatropha dioica. Journal of Natural Products, 80(8), 2252-2262. https://doi.org/10.1021/acs.jnatprod.7b00193

Pitarokili, D., Tzakou, O., Loukis, A., \& Harvala, C. (2003). Volatile metabolites from Salvia fruticosa as antifungal agents in soilborne pathogens. Journal of Agricultural and Food Chemistry, 51(11), 3294-3301. https://doi.org/10.1021/jf0211534

Salie, F., Eagles, P. F. K., \& Leng, H. M. J. (1996). Preliminary antimicrobial screening of four South African Asteraceae species. Journal of Ethnopharmacology, 52(1), 27-33. https://doi.org/10.1016/0378-8741(96)01381-5

Serrano-Gallardo, L.-B., Castillo-Maldonado, I., Borjón-Ríos, C.-G., Rivera-Guillén, M.-A., Morán-Martínez, J., Téllez- López, M.-A., ... Vega-Menchaca, M. del C. (2017). Antimicrobial activity and toxicity of plants from northern Mexico. Indian Journal of Traditional Knowledge, 16(2), 203-207.

Silva-Belmares, Y., Rivas-Morales, C., Viveros-Valdez, E., de la Cruz-Galicia, M. G., \& Carranza-Rosales, P. (2014). Antimicrobial and cytotoxic activities from Jatropha dioica roots. Pakistan Journal of Biological Sciences, 17(5), 748-750. https://doi.org/10.3923/pjbs.2014.748.750

Silva-Mares, D., Torres-López, E., Rivas-Estilla, A. M., Cordero-Pérez, P., Waksman-Minsky, N., \& Rivas-Galindo, V. M. (2013). Plants from Northeast Mexico with anti-HSV activity. Natural Product Communications, 8(3), 297-298. https://doi.org/10.1177/1934578x1300800305

Steinmann, W. V. (2002). Diversidad y endemismo de la familia Euphorbiaceae en México. Acta Botanica Mexicana, 61, 61-93.

Villarreal, A. M., Dominguez, X. A., Williams, H. J., Scott, A. I., \& Reibenspies, J. (1988). Citlalitrione, a New Diterpene from Jatropha dioica var. sessiliflora. Journal of Natural Products, 51(4), 749-753. https://doi.org/10.1021/np50058a014

Waizel-Bucay, J., \& Martínez Rico, I. (2011). Algunas plantas usadas en México en padecimientos periodontales. Revista ADM, 68(2), 73-88. 